\title{
Impact of spectral body imaging in patients suspected for occult cancer: a prospective study of 503 patients
}

\author{
Michael Brun Andersen ${ }^{1,2,3} \cdot$ Dyveke Ebbesen ${ }^{3} \cdot$ Jesper Thygesen ${ }^{4} \cdot$ Matthijs Kruis $^{5} \cdot$ Finn Rasmussen ${ }^{3}$
}

Received: 9 December 2019 / Revised: 23 March 2020 / Accepted: 8 April 2020 / Published online: 4 May 2020

(C) The Author(s) 2020

\begin{abstract}
Objectives To investigate the diagnostic impact and performance of spectral dual-layer detector $\mathrm{CT}$ in the detection and characterization of cancer compared to conventional CE-CT.

Methods In a national workup program for occult cancer, 503 patients ( 286 females and 217 males) were prospectively enrolled for a contrast-enhanced spectral CT scan. The readings were performed with and without spectral data available. A minimum of 3 months between interpretations was implemented to minimize recall bias. The sequence of reads for the individual patient was randomized. Readers were blinded for patient identifiers and clinical outcome. Two radiologists with 9 and 33 years of experience performed the readings in consensus. If disagreement, a third radiologist with 11 years of experience determined the outcome of the reading Results Significantly more cancer findings were identified on the spectral reading. In 73 cases of proven cancer, we found a sensitivity of $89 \%$ vs $77 \%$ and a specificity of $77 \%$ vs $83 \%$ on spectral CT compared to conventional CT. A slight increase in reading time in spectral images of $82 \mathrm{~s}$ was found ( $382 \mathrm{vs} 300, p<0.001$ ). For all cystic lesions, the perceived diagnostic certainty increased from $30 \%$ being completely certain to $96 \%$ most pronounced in the kidney, liver, thyroid, and ovaries. And adding the spectral information to the reading gave a decrease in follow-up examination for diagnostic certainty $(0.25$ vs 0.81 per reading, $p<0.001)$.

Conclusion The use of contrast-enhanced spectral CT increases the confidence of the radiologists in correct characterization of various lesions and minimizes the need for supplementary examinations.

Key Points

- Spectral CT is associated with a higher sensitivity, but a slightly lower specificity compared to conventional CT.

- Spectral CT increases the confidence of the radiologists.

- The need for supplementary examinations is decreased, with only a slight increase in reading times.
\end{abstract}

Keywords Helical CT $\cdot$ Clinical oncology $\cdot$ Thorax $\cdot$ Abdomen

Electronic supplementary material The online version of this article (https://doi.org/10.1007/s00330-020-06878-7) contains supplementary material, which is available to authorized users.

Michael Brun Andersen

Michael.brun.andersen@ regionh.dk

1 Department of Radiology, Copenhagen University Hospital Herlev and Gentofte, Gentofte Hospitalsvej 1, 2900 Hellerup, Denmark

2 Department of Radiology, Zealand University Hospital Roskilde, Sygehusvej 10, Roskilde 4000, Denmark

3 Department of Radiology, Aarhus University Hospital, Palle Juul-Jensens Blvd. 161, Aarhus 8200, Denmark

4 Department of Clinical Engineering, Central Denmark Region, Nørrebrogade 44, Building 2A, Aarhus 8000, Denmark

5 Philips Medical Systems, Clinical Science, CT, Veenpluis 4-6, Best 5684, The Netherlands

$\begin{array}{ll}\text { Abbreviations } \\ \text { CE-CT } & \begin{array}{l}\text { Contrast-enhanced computed tomography } \\ \text { CE-SCT }\end{array} \\ & \begin{array}{l}\text { Contrast-enhanced spectral computed } \\ \text { tomography }\end{array} \\ \text { CT } & \text { Computed tomography } \\ \text { DK } & \text { Denmark } \\ \text { DLP } & \text { Dose length product } \\ \text { GP } & \text { General practitioner } \\ \text { HU } & \text { Hounsfield units } \\ \text { keV } & \text { Kiloelectron volt } \\ \text { kVp } & \text { Kilovolt peak } \\ \text { mAs } & \text { Milliamperes per second } \\ \text { MRI } & \text { Magnetic resonance imaging } \\ \text { NBH } & \text { National Board of Health } \\ \text { ROI } & \text { Region of interest } \\ \text { VNC } & \text { Virtual non-contrast } \\ Z_{\text {eff }} & \text { Effective atomic number }\end{array}$




\section{Introduction}

Computed tomography (CT) is the leading modality for the detection and characterization of neoplasms [1]. Iodine is commonly used as a contrast agent, because it provides great $\mathrm{CT}$ contrast and can relatively safely be injected into the bloodstream [2]. The fact that perfusion is considered a hallmark of cancer biology [3] makes iodine contrast-enhanced $\mathrm{CT}$ a very potent modality for cancer diagnoses.

Developments in dual-energy or spectral CT have been a major leap for CT. Most techniques create a dual-energy dataset by scanning the same area twice with different kilovolt peak/filtration settings. This can be achieved either by performing two completely separate acquisitions, by rapidly alternating the kilovolt peak during the rotation, or by using two X-ray sources and detectors in one CT scanner. The latest commercial spectral solution uses a dual-layer detector to separate the $\mathrm{x}$-ray spectrum, without the need for changing the tube kilovolt peak or any other scanning parameters. The top row detects low-energy photons while the bottom layer detects high-energy photons [4].

Spectral CT improves quantification of materials and characterization of tissue in comparison to conventional singleenergy CT. Where conventional CT provides Hounsfield units (HUs) that describe the scanned tissues relative x-ray attenuation properties for a particular tube output spectrum, spectral $\mathrm{CT}$ provides quantitative measures like material decomposition, material concentrations, and suppression of signals associated with specific materials. There is a wide variety of materials to which this can be applied like iodine, water, calcium, uric acid, fat, and iron $[5,6]$.

Improvement of iodine quantification and visualization with spectral CT has shown improved detection and characterization of a wide variety of neoplasms [7]. Examples of these are improvements in lung nodule characterization [8], hypervascular liver lesion detection $[9,10]$, differentiation between malignant and benign thyroid nodules [11, 12], characterization of lesions as simple cysts in the kidneys [13-15], adrenal adenoma and metastasis differentiation [16, 17], hypodense pancreatic lesion delineation [18], detection of iso-attenuating pancreatic lesions $[19,20]$, and differentiation of prostate carcinoma from benign hyperplasia [21].

In Denmark (DK), the National Board of Health (NBH) has defined health packages for diagnosis and treatment of specific diseases and patient groups, to streamline diagnoses and treatment. Furthermore, it ensures that all patients are treated according to national guidelines.

Among these packages, 28 specific cancer treatment pathways are implemented. With specific cancer symptoms, patients will be directed to specific pathways. However, when a patient presents to the general practitioner with a set of serious, but more vague, cancer-indicating symptoms (e.g., fatigue, pain, fever, unintended weight loss, abnormal blood chemistry), the patient can be referred to a package for suspected serious illness that could be cancer (occult cancer package). The incidence of cancer for patients enrolled in this package lies around $16 \%$ and based on recommendation from the National Board of Health, whole body iodine contrastenhanced CT is the imaging modality of choice in such patients [22].

The purpose of this study is to evaluate the impact of contrast-enhanced spectral CT (CE-SCT) compared to contrast-enhanced CT (CE-CT) in a prospectively gathered cohort of patients that enter the occult cancer pathway. The hypothesis is that CE-SCT will detect more lesions than CE$\mathrm{CT}$, that CE-SCT is better in the characterization of a lesion than CE-CT, and that the confidence level of the reporting radiologist increases using CE-SCT compared to CE-CT. Furthermore, workflow and need for supplementary diagnostics will also be presented.

\section{Materials and methods}

\section{Patients}

Five hundred thirty-six patients, referred by their general practitioner (GP) to the occult cancer pathway, were prospectively included in the study between May 2017 and November 2018. The ethical committee waived ethical issues and the study was approved by the Danish data authorities. Five hundred three patients gave written consent to participate in the project, and allowed access to image and clinical data.

Patients referred from the GP to the fast track package for suspected serious illness that could be cancer were eligible for inclusion. Exclusion criteria were missing written consent, scan protocol differing from the national guidelines, and allergies to iodine contrast media. Figure 1 provides an overview of the study design.

Patient in the study population presented themselves to the GP with numerous symptoms and risk factors (see Table 1). Unfortunately, the referral information is often incomplete.

An overview of the basic demographics is included in Table 2.

\section{Spectral contrast-enhanced CT and contrast-enhanced CT}

SCE-CT of the chest, abdomen, and pelvis was acquired on a 64-row dual-layer detector CT scanner (Philips IQon; Philips Healthcare). CT acquisition parameters were $64 \times 0.625-\mathrm{mm}$ collimation, kilovolt peak 120-140, milliamperes per second/ slice $150-250$, rotation time $0.75 \mathrm{~s}$, reconstruction thickness $2 \mathrm{~mm}$, increment $1 \mathrm{~mm}$, pitch 1.078 , FOV $35 \mathrm{~cm}$, and matrix $512 \times 512$. Iodixanol $270 \mathrm{mg} / \mathrm{ml}$ (Visipaque ${ }^{\circledR}$ 270; GE Healthcare), or iohexol $300 \mathrm{mg} / \mathrm{ml}$ (Omnipaque ${ }^{\circledR}$ 300; GE 


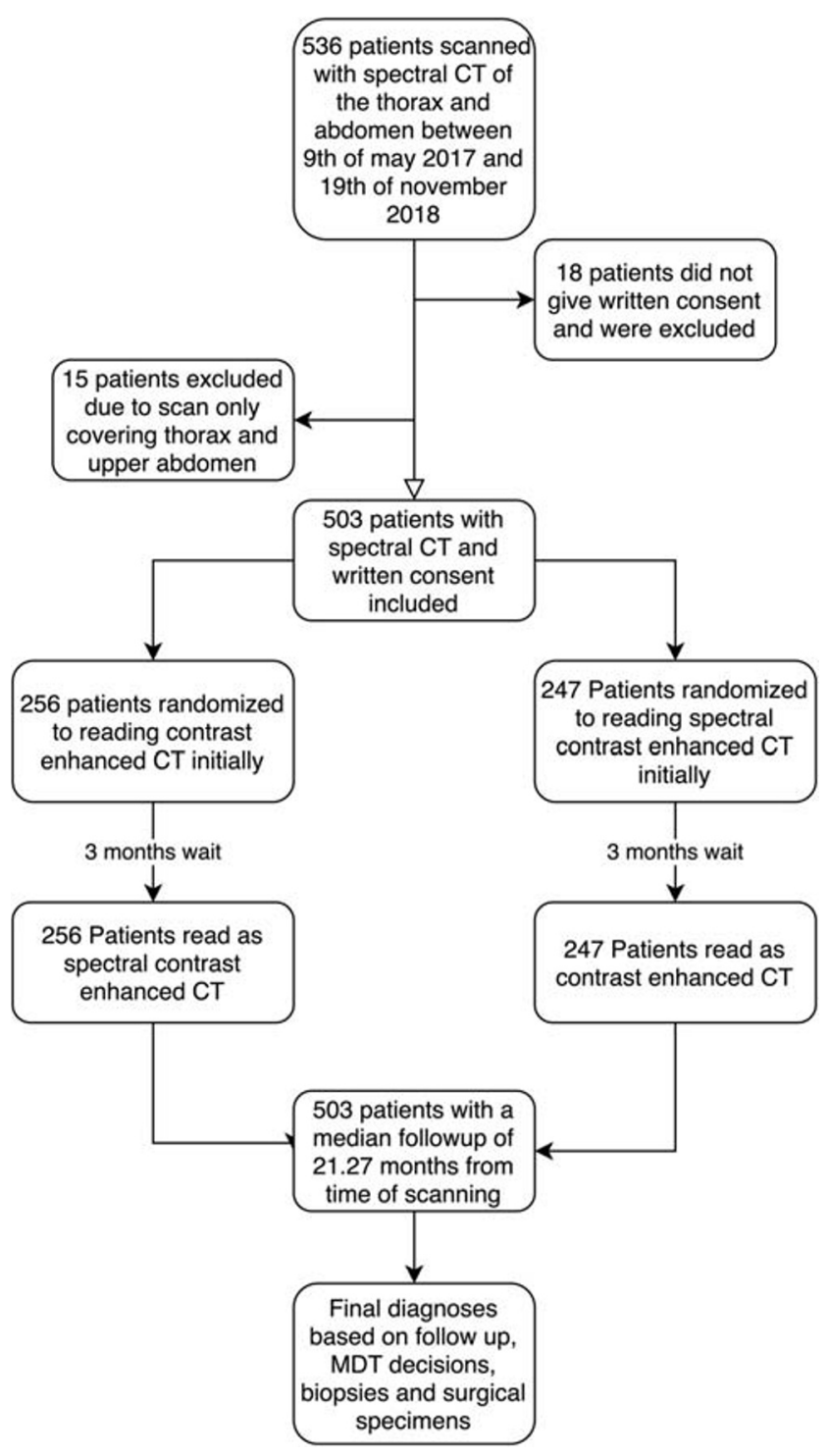

Fig. 1 A flowchart of inclusion, exclusion, and the overall study design

Healthcare), was injected intravenously in weight-adjusted doses of $2 \mathrm{ml} / \mathrm{kg}$ body weight to compensate for differences in distribution volume, with an injection rate of $4 \mathrm{ml} / \mathrm{s}$. A bolus tracking technique was used with a ROI in the descending aorta on the level of carina to compensate for differences in cardiac output. A threshold of $150 \mathrm{HU}$ was used and CT was performed after a delay of $15 \mathrm{~s}$ for the chest and upper abdomen (late arterial phase), and $65 \mathrm{~s}$ for the abdomen (portal venous phase). The mean dose length product (DLP) of CT scans performed on the population was $2104 \mathrm{mGy} \cdot \mathrm{cm}$ (CI95\% 2064 to 2144). By spectral separation of the CT signal in the two detector layers, a spectral CT dataset was reconstructed. By weighted addition of the signal of the two layers together before reconstruction, a conventional CT dataset was reconstructed that possesses all features of a normal single energy CT in terms of dose [23] and image quality [24].
Table 1 Symptoms presented by the patients to the general practitioner and used in the referral for the workup program. The list also reports the number of smokers and patients with an earlier cancer diagnosis

\begin{tabular}{lr}
\hline Symptoms/risk factors & $n$ \\
\hline Weight loss & 240 \\
Pain & 185 \\
Fatigue & 181 \\
Diarrhea & 87 \\
Dyspepsia & 73 \\
Anemia & 72 \\
Coughing & 61 \\
Nightly sweats & 59 \\
Increased liver parameters & 53 \\
Dyspnea & 49 \\
Palpable tumor & 35 \\
Increased abdominal width & 29 \\
Back pain & 14 \\
Smoking & 61 \\
Earlier diagnosed with & 60 \\
$\quad$ cancer & \\
\hline
\end{tabular}

\section{Reading of examinations}

Scan data were transferred to a dedicated spectral workstation (Intellispace 9; Philips Healthcare) and divided into primary readings and secondary reading folders. The reading folders contained either the CE-CT or a superset of the conventional results and the CE-SCT. The provided spectral data include the following: virtual monoenergetic images (ranging from 40 to $200 \mathrm{keV})$, effective atomic number $\left(Z_{\text {eff }}\right.$, reporting the atomic number of the tissue), iodine-no-water (pure spectral decomposition of iodine and water. Calcium remains visible and is mimicked by iodine), iodine density (similar to iodine no water, but calcium is masked out), contrast enhancing structures (masking of iodinated tissues), uric acid (masking of uric acid containing tissue), and virtual non-contrast (VNC, a $70 \mathrm{keV}$ map without the signal of iodine contrast).

Overlay images on the conventional series or the $\mathrm{VNC}$ was available for all datasets.

The scans were hereafter read in consensus by two experienced radiologists, with respectively 9 and 33 years of experience. In case of disagreement, a third radiologist would determine the outcome of the proposed findings.

All organ systems covered in the scans were reviewed. Initial reading was performed with virtual monoenergetic $40 \mathrm{keV}$ to identify lesions that required further attention. In suspicion of disease, other spectral results were investigated. For instance, VNC was used in the case of a suspicion of calcification to discriminate calcium from iodine. In contrary, for low- or hyperattenuated lesions, iodine density, iodine no water, contrast enhancing 
Table 2 Patient demographics: gender, age, weight, height, radiation dosage, and patient categories

\begin{tabular}{|c|c|c|c|c|c|c|}
\hline & $n$ & Mean & $\begin{array}{l}\text { Std. } \\
\text { dev. }\end{array}$ & $\begin{array}{l}95 \% \text { confidence } \\
\text { interval }\end{array}$ & Min & $\operatorname{Max}$ \\
\hline Males & 216 & & & & & \\
\hline Females & 287 & & & & & \\
\hline Age (year) & 503 & 64.48 & 13.20 & $53.29-65.67$ & 23 & 90 \\
\hline Height (cm) & 467 & 171.61 & 8.80 & $170.81-172.41$ & 147 & 196 \\
\hline Weight $(\mathrm{kg})$ & 469 & 73.53 & 15.64 & $72.11-74.95$ & 33 & 150 \\
\hline Dose length product $(\mathrm{mGy} \times \mathrm{cm})$ & 503 & 2104.12 & 441.91 & $2064.24-2144.00$ & 1375.9 & 4778 \\
\hline Proven cancer & 73 & & & & & \\
\hline Likely cancer based on imaging & 15 & & & & & \\
\hline Unlikely cancer & 31 & & & & & \\
\hline Other proven serious disease & 22 & & & & & \\
\hline Proven benign & 152 & & & & & \\
\hline $\begin{array}{l}\text { No finding described in } \\
\text { follow-up }\end{array}$ & 210 & & & & & \\
\hline
\end{tabular}

structures, and $Z_{\text {eff }}$ were used to prove the presence of iodine.

To prevent recall bias, the primary and secondary reading were performed with an interval of at least 3 months and in random order. The radiologists were blinded from patient identifiers and earlier imaging, but had access to symptoms and a concise medical background of the patient mentioned in the referral.

All findings were entered into a RedCAP database [25]. Up to 7 findings per patients were classified according to their severity and were scored for malignancy of the finding $(1=$ "certainly malignant," 2 = "probably malignant," 3 = "probably benign," and $4=$ "certainly benign") and certainty of the finding $(1=$ "certain," 2 = "almost certain," 3 = "somewhat uncertain," and $4=$ "very uncertain").

During the course of the study, we decided to record additional information. For a subset of the readings ( 308 spectral and 304 conventional), we recorded the reading time. For another subset (418 spectral and 414 conventional), we recorded the need for supplementary examinations. For the last 221 patients (221 spectral and 221 conventional readings), we also recorded whether the indicated supplementary examinations were part of routine or needed to improve the certainty of the finding.

Routine follow-up procedures included a wide range of examinations, including CT thorax in case of pulmonary nodules according to Fleischner Society criteria, multiphase CT or ultrasound in case of hyperdense lesions in the kidney, PET/CT in the case of suspected lung cancer, transvaginal ultrasound for ovarian lesions, dedicated adrenal CT for adrenal lesions, ultrasound and possible biopsy from thyroid lesions, etc. In the case of uncertainty, the follow-up procedures include contrastenhanced ultrasound in liver or kidney lesions, biopsy or follow-up on enlarged/suspicious lymph nodes, endoscopies when intraluminal lesions, or mural thickening was suspected etc.

\section{Review of findings}

Individual findings were matched between the two readings, according to disease type and associated organ.

After a median follow-up of 21.3 months, the clinical status was recorded for every patient. When the clinical status could not validate a recorded serious first finding (cancer or another disease that would need immediate medical treatment), a more thorough search in the medical records was conducted for validation. When a serious finding was not considered in the medical history of the patient, the case was reported to the Ethical Committee for additional follow-up.

Every patient would now have an outcome value $(1=$ "cancer, with recorded proof," 2 = "likely cancer, without recorded proof," 3 = "unlikely cancer," $4=$ "proven benign," $1=$ "finding not investigated," $\mathrm{O}=$ "other proven serious disease").

Most of the benign findings were not reported in the patient dossier and would get a score of " -1 ." There were also potential malignant findings without follow-up that received a score of "2" or "3." Very unlikely cancers were often not investigated and patients often did not want follow-up diagnostics or were not able to cooperate/receive the suggested procedure. Other serious diseases ("O") were conditions that required treatment within a short period of time.

Cancer, with recorded proof, was obtained by the following methods: biopsy in 48 cases, surgery in 9 cases, multidisciplinary team decision in 11 cases, follow-up in 2 cases, and in 2 cases the patient had a diagnosed cancer not reported in the initial referral. 


\section{Statistical analysis}

We hypothesized that CE-SCT would detect more findings than CE-CT. We used McNemar's test to identify types of findings that differed in frequency between the two modalities.

To test the hypothesis that spectral findings provide a higher confidence to the radiologist in the diagnoses compared to conventional findings, we used a two-sided two proportion $z$-test to find the types of findings for which the proportion of certain and uncertain findings differed between CESCT and CE-CT.

We compared sensitivity and specificity of malign findings between CE-SCT and CE-CT and tested for significance by means of McNemar's test.

We performed descriptive statistics and box-and-whisker plots for the reading time per patient of both CE-SCT and CE-CT. Differences in reading time between CE-SCT and CE-CT based on the certainty of the first finding were also compared using a box-and-whisker plot.

Furthermore, we compared the frequency of supplemental procedures between CE-SCT and CE-CT by means of a twosided $t$ test.

\section{Results}

There were slightly more findings reported on the spectral than the conventional readings (6.8 vs. 6.4 findings per patient, $p<0.001)$. An overview of specific diseases with significantly different frequencies can be found in Table 3 .

Table 4 lists the frequencies of various types of findings with significant differences in certainty.

In the patient population, 73 proven cancers were observed together with 22 other serious diseases (Table 2). Imaging characteristics for the proven cancers are shown in Table 5. The sensitivity for finding these 73 cancer cases was higher in the spectral than in the conventional readings ( $89 \%$ vs. $77 \%$, $p<0.005$ ).

The specificity of the cancer findings was slightly smaller in the spectral findings ( $77 \%$ vs. $83 \%, p<0.005)$. The main reason for this difference was 18 possible prostate cancer findings without follow-up. We found a lower positive predictive value ( $40 \%$ vs $43 \%$ ), but a higher negative predictive value (98\% vs $96 \%$ ) for CE-SCT.

The reading time differed significantly between the spectral readings (see Electronic Supplementary Material). The radiologists spent on average $382 \mathrm{~s}$ analyzing the spectral readings versus $300 \mathrm{~s}$ at the conventional readings. The difference in reading time between the spectral and the conventional reading was largest when the first finding of the spectral reading was uncertain. When the first finding on the spectral reading was certain, the difference was much smaller.
Although more findings were defined during the spectral reading, we found a significantly lower number of requested follow-up procedures per reading (for spectral 0.91 and for conventional 1.39 follow-ups per reading, $p<0.001$ ). For the last 221 patients, we also distinguished between followups for clinical routine and follow-ups needed for diagnosis certainty. We found a decrease in the need for follow-ups for diagnosis certainty $(0.25$ vs. 0.81 follow-ups per reading, $p<0.001)$. The numbers of clinical routine follow-ups did not differ significantly between the two readings $(0.62 \mathrm{vs}$. $0.66, p=0.43)$.

\section{Discussion}

Godfrey Hounsfield proposed the concept of using multiple energies in computed tomography to perform material decomposition already in 1973 [26]. However, the first clinical CT scanner that could perform both dual-energy series in one rotation was developed in 2008 as a dual source system [27]. Although many studies have indicated a clinical advantage in a wide variety of applications, clinical adaptation has been lacking due to an associated increase in reading time, additional data, and reconstruction time compared to the CECT [28].

In the early iterations of dual-energy $\mathrm{CT} /$ spectral $\mathrm{CT}$, increased radiation dose was a major concern [29]. With the current scanner technology, the dose has been decreased and the concern is outweighed by the benefits associated with spectral CT [30]. In this study, the mean dose measured by DLP was $2104 \mathrm{mGy} \cdot \mathrm{cm}$ (CI95\% 2064 to 2144, range from 1081 to 4778 ). A total of 28 patients had a BMI of $<18.5$ (mean 24.9 CI95\% 24.5 to 25.3, range from 13.2 to 55.1 ) and were considered underweight. In a standard setting with CE-CT, they would most likely have been scanned with $100 \mathrm{kVp}$; however, none of the present dual-energy/spectral CT scanners are capable of producing images of diagnostic quality and provide dual-energy information at doses comparable to single-energy $100-\mathrm{kVp}$ images. It corresponds to roughly $5 \%$ of the population described in our study and in our opinion the benefits outweigh the minor increase in average patient dose.

In this study, a scanner with a dual-layer detector that is able to perform spectral acquisitions, without changing the acquisition protocol, was used. The reconstruction of the Spectral Base Images took typically less than $5 \mathrm{~min}$, after which all spectral results were instantly available to the radiologist, without the need for additional reconstructions at the workstation.

Due to this integrated solution, the additional mean spectral reading time was limited to an increase of $82 \mathrm{~s}$ compared to CE-CT (see Electronic Supplementary Material). It is important to acknowledge that these reading times are associated to 
Table 3 Organs/findings with a significant difference in incidence between the conventional contrast-enhanced CT and the spectral contrast-enhanced CT

\begin{tabular}{|c|c|c|c|c|c|c|}
\hline Organ & Description of finding & $\begin{array}{l}\text { Found on both } \\
\text { modalities }\end{array}$ & $\begin{array}{l}\text { Number of patients } \\
\text { with no finding }\end{array}$ & $\begin{array}{l}\text { Found on } \\
\text { SCE-CT }\end{array}$ & $\begin{array}{l}\text { Found on } \\
\text { CCE-CT }\end{array}$ & $\begin{array}{l}p \text { value } \\
\text { McNemar's test }\end{array}$ \\
\hline \multicolumn{7}{|c|}{ Pancreas } \\
\hline & Cancer/tumor & 8 & 489 & 6 & 0 & 0.04 \\
\hline \multicolumn{7}{|c|}{ Prostate } \\
\hline & Process/localized enhancement & 3 & 443 & 57 & 0 & $<0.001$ \\
\hline & Hypertrophy & 44 & 397 & 12 & 50 & $<0.001$ \\
\hline \multicolumn{7}{|c|}{ Adrenal } \\
\hline & Adenoma & 1 & 473 & 28 & 1 & $<0.001$ \\
\hline & Incidentaloma & 10 & 449 & 10 & 34 & $<0.001$ \\
\hline \multicolumn{7}{|c|}{ Thyroid } \\
\hline & All findings (cysts, nodules, etc.) & 86 & 353 & 44 & 20 & $<0.001$ \\
\hline & Thyroid nodule & 13 & 440 & 39 & 11 & $<0.001$ \\
\hline \multicolumn{7}{|l|}{ Biliary } \\
\hline & All findings (stones, polyps, etc.) & 63 & 403 & 26 & 11 & 0.02 \\
\hline & Polyp & 0 & 493 & 10 & 0 & $<0.001$ \\
\hline \multicolumn{7}{|l|}{ Heart } \\
\hline & Coronary calcification & 39 & 359 & 88 & 17 & $<0.001$ \\
\hline
\end{tabular}

the specific system, workflow, and setup at our institution and that it was done in a research setting with no distractions. Reading times might differ with other dual-energy CT/ spectral CT systems and institutional setups, and should be investigated thoroughly.

Numerous publications on the use of spectral CT for the detection and characterization of neoplasms have been published over the past decade. Most publications only include small and selected patient populations [28]. The aim of this study was to implement current knowledge into a daily clinical routine on a patient population with a high probability of cancer. The major outcome of this study was a higher observed sensitivity of CE-SCT compared to CE-CT when malignancy is considered ( $89 \%$ vs. $77 \%, p<0.005)$, albeit with a slightly lower observed specificity $(77 \%$ vs. $83 \%, p<0.005)$. The main reason for this decrease in specificity was prostate

Table 4 The frequency of various findings with significantly differences in certainty, as indicated by the radiologists in the readings

\begin{tabular}{|c|c|c|c|c|c|c|}
\hline Finding type & Location of finding & $\begin{array}{l}\text { Completely certain } \\
\text { SCE-CT }\end{array}$ & $\begin{array}{l}\text { Uncertain } \\
\text { SCE-CT }\end{array}$ & $\begin{array}{l}\text { Completely certain } \\
\text { CCE-CT }\end{array}$ & $\begin{array}{l}\text { Uncertain } \\
\text { CCE-CT }\end{array}$ & $p$ value $Z$ test \\
\hline \multirow[t]{5}{*}{ All findings } & Overall & 2415 & 158 & 1920 & 522 & $<0.001$ \\
\hline & Kidney & 271 & 12 & 117 & 153 & $<0.001$ \\
\hline & Liver & 269 & 30 & 126 & 164 & $<0.001$ \\
\hline & Thyroid & 134 & 5 & 54 & 46 & $<0.001$ \\
\hline & Adrenal & 56 & 0 & 49 & 6 & 0.011 \\
\hline \multirow[t]{5}{*}{ Cysts } & Overall & 474 & 20 & 142 & 331 & $<0.001$ \\
\hline & Kidney & 203 & 7 & 60 & 141 & $<0.001$ \\
\hline & Liver & 149 & 6 & 26 & 139 & $<0.001$ \\
\hline & Thyroid & 65 & 1 & 23 & 32 & $<0.001$ \\
\hline & Ovaries & 28 & 1 & 19 & 5 & 0.047 \\
\hline \multirow{4}{*}{$\begin{array}{l}\text { Process (tumor, nodule, } \\
\text { neoplasm) }\end{array}$} & Overall & 136 & 16 & 56 & 25 & $<0.001$ \\
\hline & Liver & 20 & 5 & 8 & 8 & 0.044 \\
\hline & Thyroid & 37 & 2 & 12 & 11 & $<0.001$ \\
\hline & Prostate & 52 & 3 & 0 & 1 & $<0.001$ \\
\hline Calcifications & Coronary vessels & 109 & 0 & 31 & 10 & $<0.001$ \\
\hline
\end{tabular}




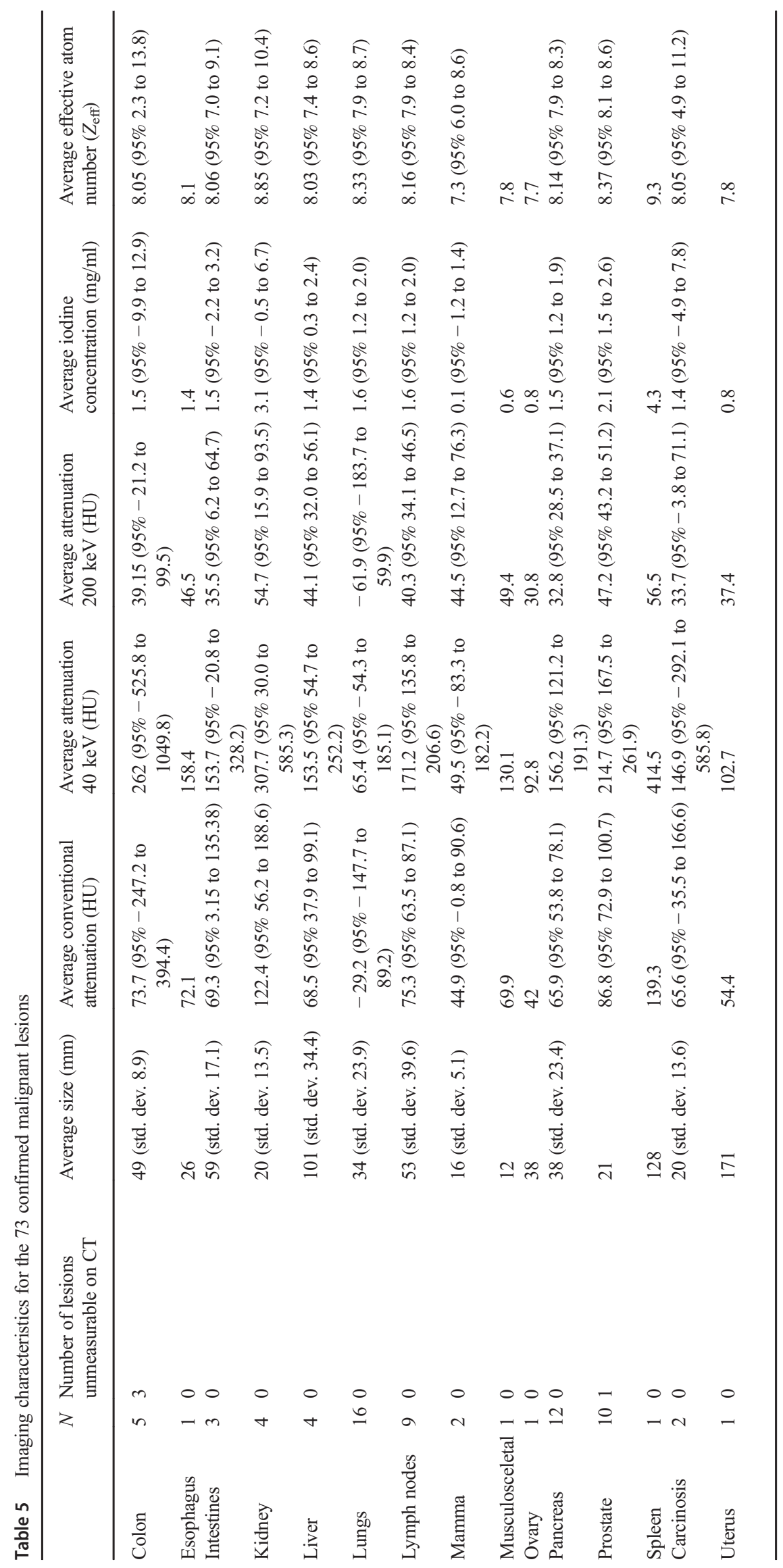


a

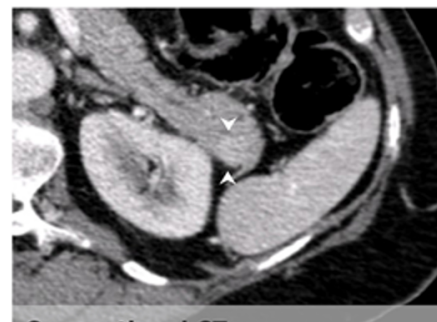

Conventional CT

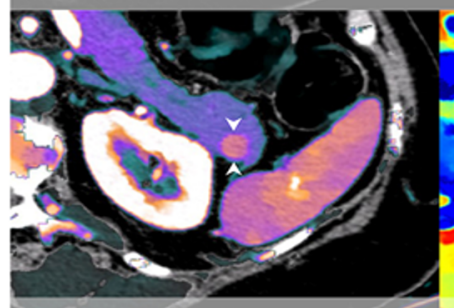

Iodine density overlay

\section{b}

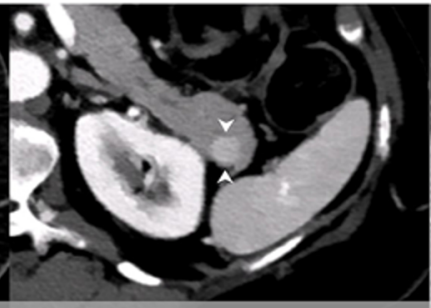

Monoenergetic $40 \mathrm{KeV}$

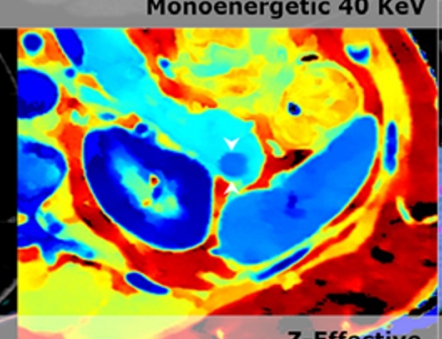

Z-Effective

Fig. 2 a In the pancreatic tail, a small lesion (arrowhead) is barely visible on the conventional CT. However, on all spectral series, the lesion becomes evident. The enhancement corresponds to the spleen and differential diagnoses were both an intrapancreatic accessory spleen and a small neuroendocrine tumor. Historic data of the patient however demonstrated that the lesion had not changed in 12 years, and therefore this lesion was characterized as an intrapancreatic accessory spleen. b A

findings, of which many were not further investigated. The increase in sensitivity and corresponding drop in specificity can somewhat be compared to other imaging modalities like positron emission tomography/CT (PET/CT), which also have a high sensitivity at the cost of an increased number of false positive findings.

In this study, we have 8 false negatives for CE-SCT comprised of 2 colon cancers, 2 pancreatic cancers, and 2 prostate cancers, even retrospectively 4 of the lesions are not found. The remaining 2 lesions were detected, but categorized as benign: a ground-glass lung nodule, followed up correctly, and a cancer mamma with no enhancement on iodine quantification.

The perceived certainty in diagnoses, especially for benign cystic lesions, increased from $30 \%$ being certain to $96 \%$, and adding the spectral information to the reading will allow a decrease in total number of follow-up examinations from 1.39 to 0.91 per exam.

The differences in sensitivity, specificity, and general certainty were concentrated within a number of specific organs: pancreas, prostate, thyroid, kidney, and adrenal glands.

Virtual low monoenergetic reconstructions increase the ability to detect and stage pancreatic cancer. In recent studies, optimal contrast-to-noise ratio settings have been determined to be $40 \mathrm{keV}$ and optimal image quality at $55-65 \mathrm{keV}$ [18]. By using these settings as part of our routine assessment of the pancreas in CE-SCT series, we found significantly more suspicious lesions in the pancreas than on CE-CT (14 vs. 8). Because the readers were blinded to prior examinations, some

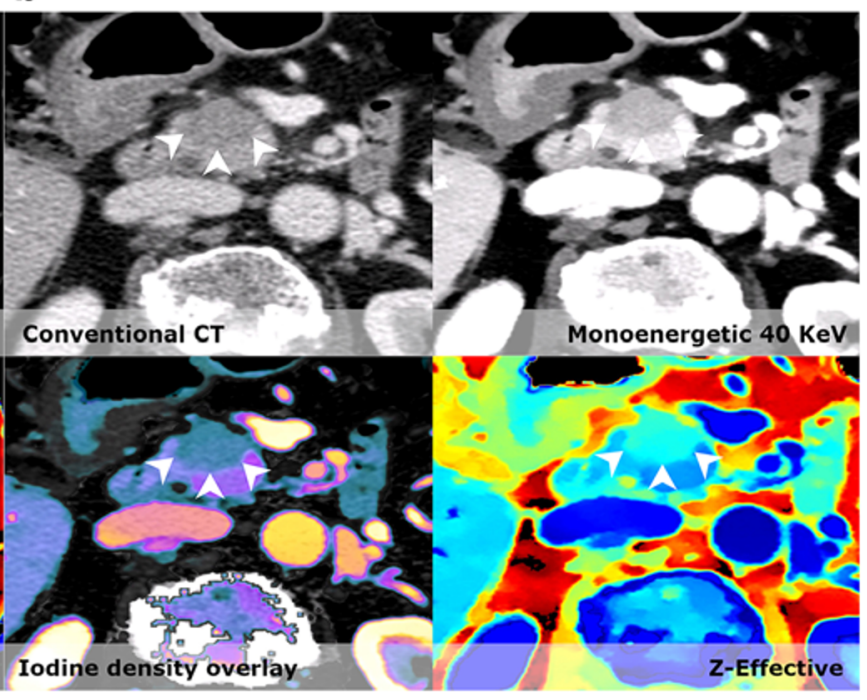

patient with a slight increase in volume in the head of the pancreas and stranding in the peripancreatic fat. In the pancreatic head, a slight hypodense lesion (arrowheads outline the lesion) is visible on the conventional image; however, it is easily missed. On all spectral images, the lesion stands out against the normal pancreatic parenchyma and the lesion was correctly diagnosed as a pancreatic adenocarcinoma

lesions found on spectral were wrongly perceived as being potentially malignant. An example of this was a case showing a roughly $1.3-\mathrm{cm}$ vague hyperdense lesion in the pancreatic tail that was missed on the conventional series (Fig. 2a). Based on the available imaging, it could represent both an intrapancreatic accessory spleen and a pancreatic neuroendocrine tumor. However, historic records of this patient described this as an intrapancreatic spleen and this lesion had been stable for over 5 years. In another patient, a definitive malignant lesion was suspected on the conventional series but became evident on CE-SCT series (Fig. 2b); the lesion later proved to be a small adenocarcinoma.

For prostate imaging, MRI is currently the imaging modality of choice. However, studies on prostate CE-CT have suggested that areas of mass-like enhancement in the periphery of the prostate on venous phase may correlate with neoplasms and should warrant further follow-up [21]. In our patient cohort, the contrast enhancement in the periphery is potentiated by using virtual low monoenergetic images. We found significantly more focal lesions within the prostate using CE-SCT compared to CE-CT. The reason for finding significantly more hypertrophic prostates on CE-CT is that in case of focal enhancement, prostate hypertrophy was not reported (Fig. 3). Unfortunately, the diagnostic impact of these changes seen on CE-SCT in the prostate gland could not be validated in the current setup. Comparative studies between CE-SCT with state-of-the-art MRI of the prostate are needed [31, 32].

Adrenal gland lesions most often represent benign adenomas, but the presence of iodine makes differentiation a 
Fig. 3 Small lesions, like this small lenticular lesion (arrow) $<1.5 \mathrm{~cm}$ in size, are often overlooked. The lesion is easy to see on both virtual low monoenergetic images, iodine density overlays, and Z-effective. It is anteriorly located on the edge of the transition zone. A biopsy confirmed that this lesion was a prostate cancer

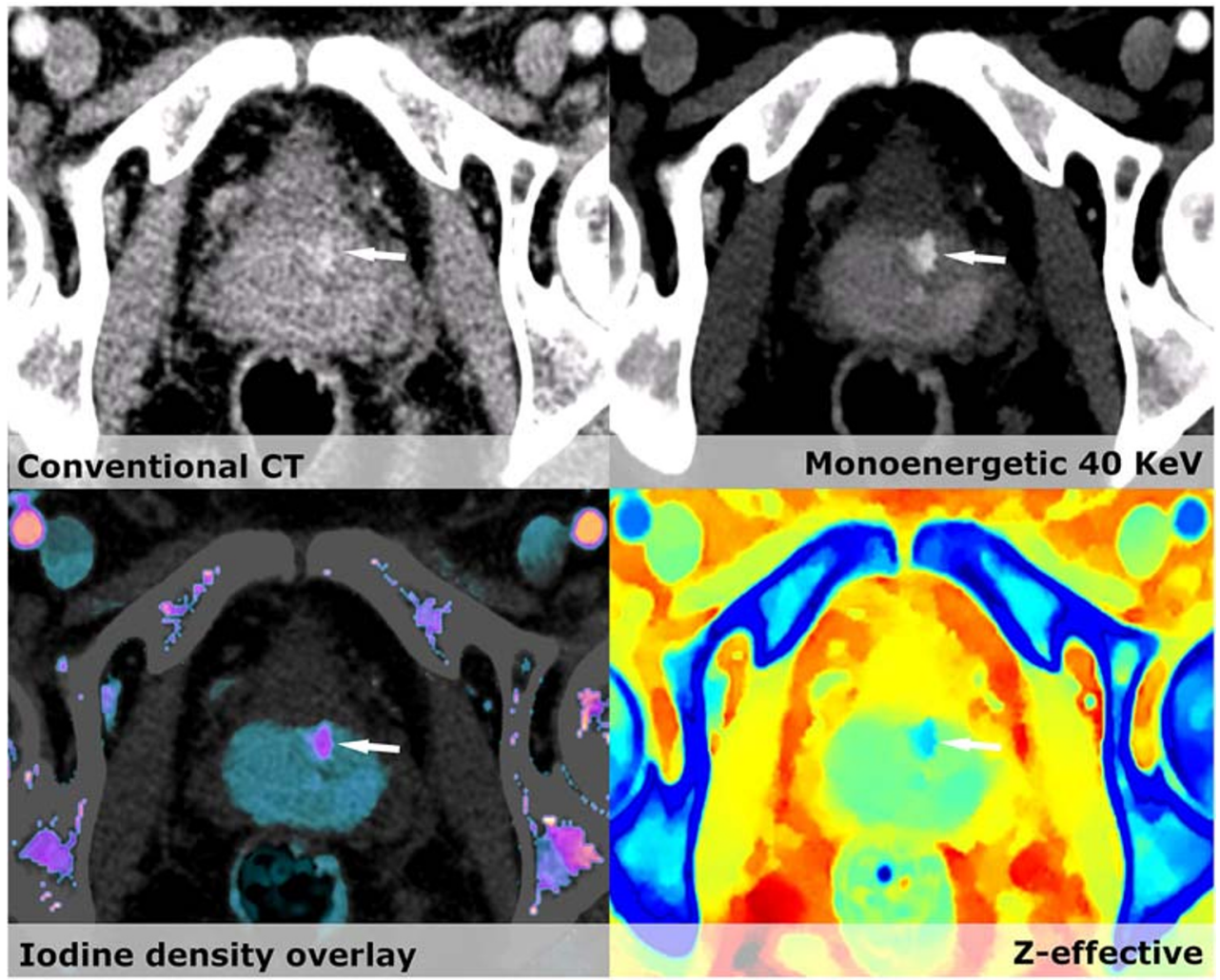

challenge on CE-CT. From earlier studies [16, 17], we know that adrenal adenomas can be confidently identified on VNC by using a cutoff value of $<10 \mathrm{HU}$ with a relatively high sensitivity of $73 \%$ and a specificity of $100 \%$. This made it
Fig. 4 On all images, a small lesion (Arrow) in the left thyroid lobe is seen. However, using the spectral information, the lesion is most likely benign with high iodine density of $>6 \mathrm{mg} / \mathrm{ml}$
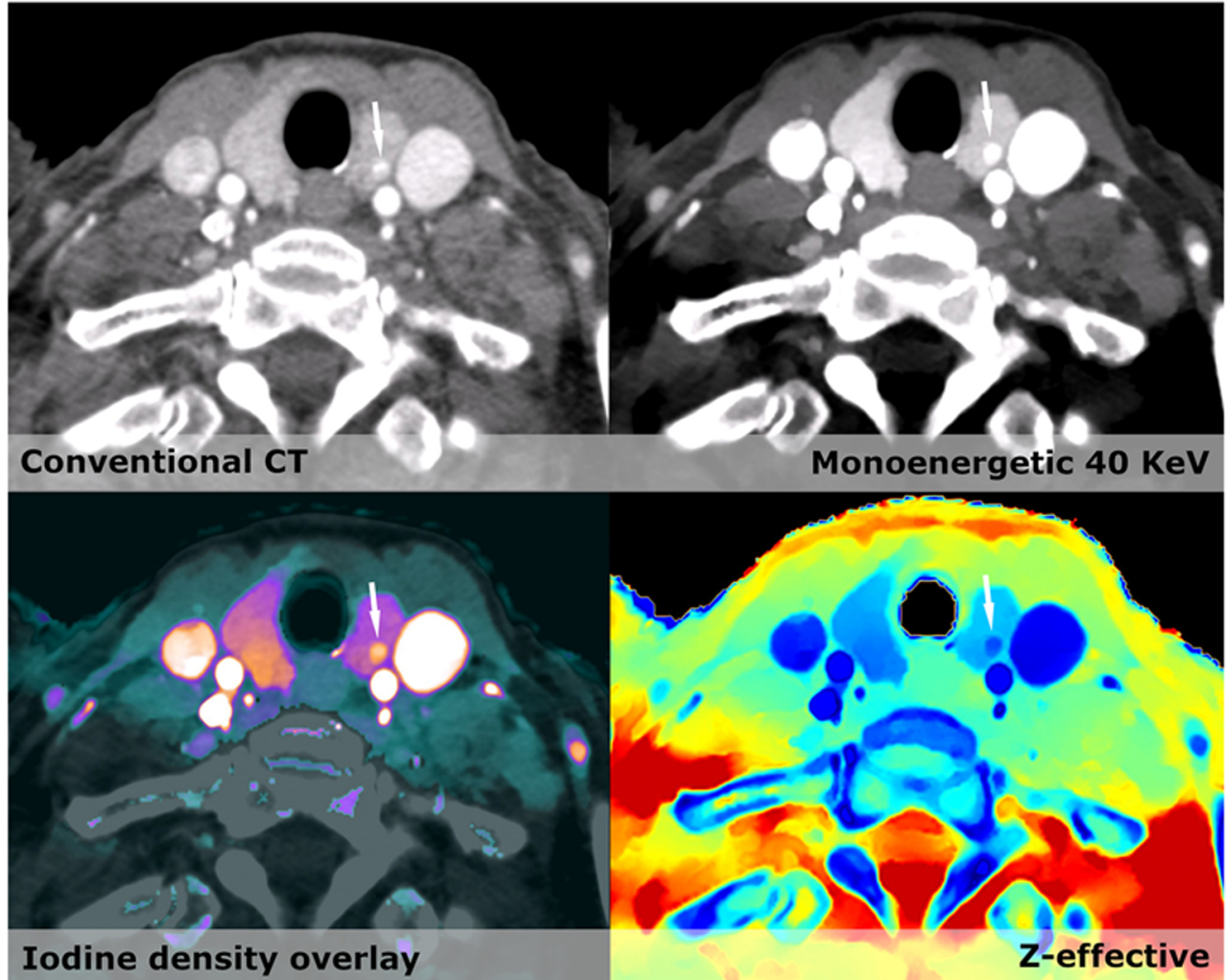
possible in the current cohort to evaluate enlarged adrenal glands and confidently characterize them in a majority of the cases, minimizing the need for added follow-up examinations. We found significantly more adrenal adenomas with CE-SCT, which related to an increase of adrenal incidentalomas found on CE-CT $[16,17]$.

Management of thyroid nodules on CE-CT has always been challenging. One study found an increase in iodine concentration and $Z_{\text {eff }}$ was able to distinguish papillary carcinomas from benign multinodular goiter [11, 12]. We observe significantly more lesions by CE-SCT and can better characterize actual iodine enhancement within them using iodine density and $Z_{\text {eff }}$ (Fig. 4). However, the evidence is scarce and will require a study design constructed to distinguish between benign and suspicious lesions.

Incidental renal masses are very common and difficult to assess without a non-contrast CT. Effective atomic number $\left(Z_{\text {eff }}\right)$ using a cutoff value of 8.36 can be used to distinguish between true enhancing and non-enhancing renal masses [10]. Even though we did not find significantly more enhancing masses in the kidneys on the CE-SCT compared to the CE$\mathrm{CT}$, the confidence level of our diagnoses of cysts was dramatically increased, therein also minimizing the need for supplementary examinations. In a case of polycystic kidney disease, CE-SCT made the reading markedly easier as it could readily distinguish between true enhancement, calcifications, hyperdense cysts, and simple cysts. CE-SCT showed a hyperdense cyst and a contrast enhancing tumor within the left kidney. CECT merely shows two hyperdense lesions (Fig. 5).

Virtual low monoenergetic series are efficient at detecting gall stones [33], as even cholesterol stones are detected (Fig. 6a). However, it may be difficult to distinguish between some types of gall stones and enhancing polyps on CE-CT. We found significantly more gall bladder polyps by relying on iodine perfusion instead of HU values. To our knowledge, the use of CE-SCT to identify gall bladder polyps has not yet been described in the literature (Fig. 6b).

When CE-SCT are considered, one shall be aware of artifacts that could affect material decomposition, especially in the vicinity of high-density materials in the form of metal implants or high concentration of iodine contrast. These can result in photon starvation leading to a deterioration of image quality on both CE-CT and CE-SCT. An example of such image deterioration can be seen in the electronic supplementary material.

The increase in reading time seems to be very well justified by the improvement in accuracy, confidence level, and a decreased need for follow-up investigations. These improvements will likely lead to a decrease in time to treat, improved patient satisfaction, and general outcome.
Fig. 5 Conventional CT shows several hyperdense lesions within the kidney besides the simply cysts. VNC shows differences in several areas, most markedly within one of the two hyperdense lesions (Arrow) in the lateral part of the kidney. Iodine density overlay and Z-effective show a clear enhancement in the area mentioned. Next to this is a hyperdense cyst (arrowhead) with no enhancement and Z-effective values below 8 . The diagnosis are most likely renal carcinoma within a polycystic kidney

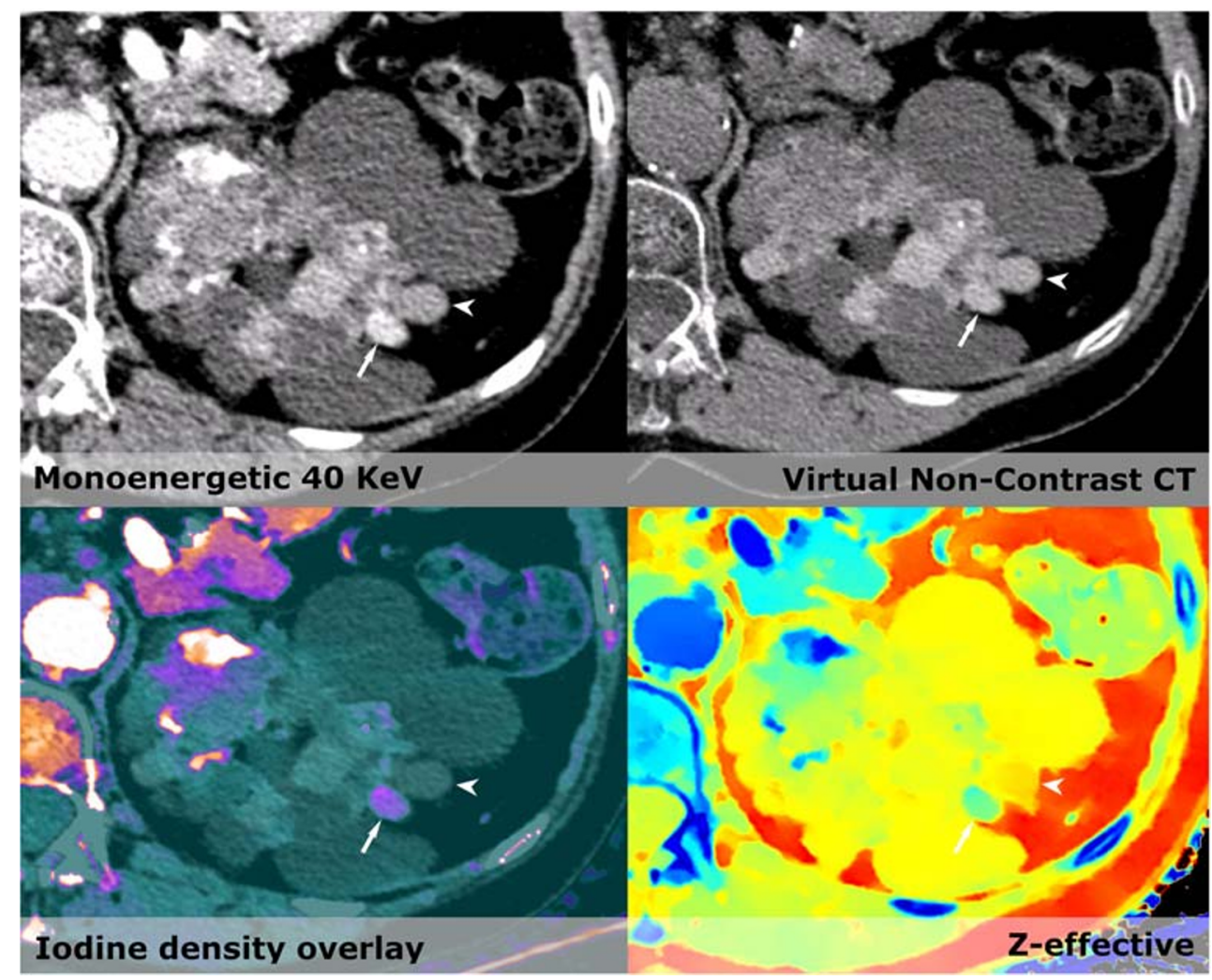


a

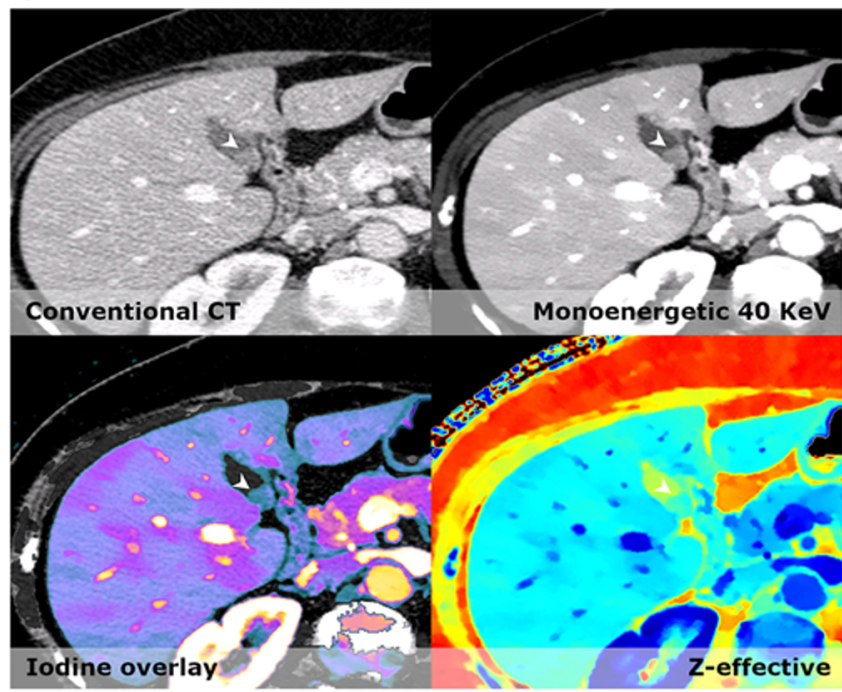

Fig. 6 a Conventional CT shows a hyperdense process (arrowhead) in the gall bladder. The virtual low monoenergetic image increases the HU of the process; iodine density overlay shows increased levels of iodine around $1 \mathrm{mg} / \mathrm{ml}$; $Z$-effective shows average atomic number close to the liver parenchyma. It could be an adenoma or adenocarcinoma; however,

Our study has some limitations. First and foremost, we lack histopathological confirmation of some diagnoses; however, for all patients, we have a relatively long median follow-up period of 21.3 months. Inter-observer variability was not performed as the study design was intended to mimic clinical routine as close as possible.

In conclusion, the use of CE-SCT on patients suspected for serious illness that could be cancer increases the confidence of the radiologists in the correct characterization of hypo- and hyperdense lesions in the liver, pancreas, and kidneys; minimizes the need for supplementary examinations at the cost of a minimal increase in reading time; but does not affect the scanner unit workflow dramatically.

Funding information The department has a general research agreement with Philips.

\section{Compliance with ethical standards}

Guarantor The scientific guarantor of this publication is Finn Rasmussen.

Conflict of interest One author (Matthijs Kruis) of this manuscript declares relationships with the following companies: Philips.

Statistics and biometry No complex statistical methods were necessary for this paper.

Informed consent Written informed consent was obtained from all subjects (patients) in this study.

Ethical approval Institutional review board approval was waived if all subjects (patients) gave written informed consent.

\section{b}

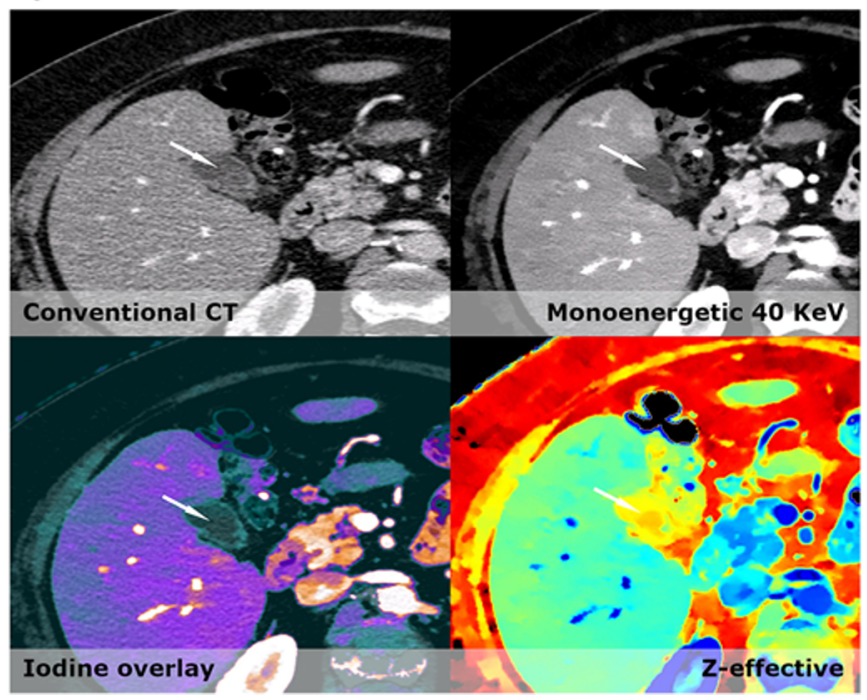

the patient did not wish to have further workup done. b Conventional CT show a slight hyperdense process in the gallbladder. Virtual low monoenergetic images make the lesion become hypodense, no iodine density is seen and $Z$-effective images show average atomic numbers closer to fat, giving us the diagnosis of a cholesterol stone

Study subjects or cohorts overlap Some study subjects or cohorts have been previously reported at ECR 2019 under control number 19-P-2344ECR.

Methodology

- Prospective

- Observational

- Performed at one institution

Open Access This article is licensed under a Creative Commons Attribution 4.0 International License, which permits use, sharing, adaptation, distribution and reproduction in any medium or format, as long as you give appropriate credit to the original author(s) and the source, provide a link to the Creative Commons licence, and indicate if changes were made. The images or other third party material in this article are included in the article's Creative Commons licence, unless indicated otherwise in a credit line to the material. If material is not included in the article's Creative Commons licence and your intended use is not permitted by statutory regulation or exceeds the permitted use, you will need to obtain permission directly from the copyright holder. To view a copy of this licence, visit http://creativecommons.org/licenses/by/4.0/.

\section{References}

1. Petralia G, Bonello L, Viotti S et al (2010) CT perfusion in oncology: how to do it. Cancer Imaging 10:8-19. https://doi.org/10. 1102/1470-7330.2010.0001

2. Rutten A, Prokop M (2007) Contrast agents in X-ray computed tomography and its applications in oncology. Anticancer Agents Med Chem 7:307-316. https://doi.org/10.2174/ 187152007780618162

3. Hanahan D, Weinberg RA (2011) Hallmarks of cancer: the next generation. Cell 144:646-674. https://doi.org/10.1016/j.cell.2011. 02.013 
4. McCollough CH, Leng S, Yu L, Fletcher JG (2015) Dual- and multi-energy CT: principles, technical approaches, and clinical applications. Radiology 276:637-653. https://doi.org/10.1148/radiol. 2015142631

5. Fredenberg E (2018) Spectral and dual-energy X-ray imaging for medical applications. Nucl Instrum Methods Phys Res, Sect A 878: 74-87. https://doi.org/10.1016/j.nima.2017.07.044

6. Johnson TR, Krauss B, Sedlmair M et al (2007) Material differentiation by dual energy CT: initial experience. Eur Radiol 17:15101517. https://doi.org/10.1007/s00330-006-0517-6

7. Simons D, Kachelriess M, Schlemmer HP (2014) Eur Radiol 24: 930-939. https://doi.org/10.1007/s00330-013-3087-4

8. Chae EJ, Song J-W, Seo JB et al (2008) Clinical utility of dualenergy CT in the evaluation of solitary pulmonary nodules: initial experience. Radiology 249:671-681. https://doi.org/10.1148/ radiol.2492071956

9. Shuman WP, Green DE, Busey JM et al (2014) Dual-energy liver $\mathrm{CT}$ : effect of monochromatic imaging on lesion detection, conspicuity, and contrast-to-noise ratio of hypervascular lesions on late arterial phase. Am J Roentgenol 203:601-606. https://doi.org/10. 2214/AJR.13.11337

10. Graser A, Johnson TRC, Chandarana H et al (2009) Dual energy CT: preliminary observations and potential clinical applications in the abdomen. Eur Radiol 19:13-23. https://doi.org/10.1007/s00330-008-1122-7

11. Li M, Zheng X, Li J et al (2012) Dual-energy computed tomography imaging of thyroid nodule specimens: comparison with pathologic findings. Invest Radiol 47:58-64. https://doi.org/10.1097/ RLI.0b013e318229fef3

12. May MS, Wiesmueller M, Heiss R et al (2019) Comparison of dualenergy CT in head and neck imaging. Eur Radiol 29:4207-4214. https://doi.org/10.1007/s00330-018-5762-y

13. Graser A, Johnson TRC, Hecht EM et al (2009) Dual-energy CT in patients suspected of having renal masses: can virtual nonenhanced images replace true nonenhanced images? Radiology 252:433-440. https://doi.org/10.1148/radiol.2522080557

14. Mileto A, Sofue K, Marin D (2016) Imaging the renal lesion with dual-energy CT and multi-energy applications in clinical practice: what can it truly do for you. Eur Radiol 26:3677-3690

15. Mileto A, Allen BC, Pietryga JA et al (2017) Characterization of incidental renal mass with dual-energy CT: diagnostic accuracy of effective atomic number maps for discriminating nonenhancing cysts from enhancing masses. Am J Roentgenol 209:W221W230. https://doi.org/10.2214/AJR.16.17325

16. Helck A, Hummel N, Meinel FG et al (2014) Can single-phase dual-energy CT reliably identify adrenal adenomas? Eur Radiol 24:1636-1642. https://doi.org/10.1007/s00330-014-3192-z

17. Connolly MJ, McInnes MDF, El-Khodary M et al (2017) Diagnostic accuracy of virtual non-contrast enhanced dual-energy CT for diagnosis of adrenal adenoma: a systematic review and metaanalysis. Eur Radiol 27:4324-4335. https://doi.org/10.1007/ s00330-017-4785-0

18. Patel BN, Thomas JV, Lockhart ME et al (2013) Single-source dual-energy spectral multidetector CT of pancreatic adenocarcinoma: optimization of energy level viewing significantly increases lesion contrast. Clin Radiol 68:148-154. https://doi.org/10.1016/j. crad.2012.06.108

19. Lin XZ, Wu ZY, Tao R et al (2012) Dual energy spectral CT imaging of insulinoma - value in preoperative diagnosis compared with conventional multi-detector CT. Eur J Radiol 81:2487-2494. https://doi.org/10.1016/j.ejrad.2011.10.028
20. Nagayama Y, Tanoue S, Oda S et al (2019) Dual-layer spectral CT improves image quality of multiphasic pancreas $\mathrm{CT}$ in patients with pancreatic ductal adenocarcinoma. Eur Radiol 394-403. https://doi. org/10.1007/s00330-019-06337-y

21. Zhang XF, Lu Q, Wu LM et al (2013) Quantitative iodine-based material decomposition images with spectral CT imaging for differentiating prostatic carcinoma from benign prostatic hyperplasia. Acad Radiol 20:947-956. https://doi.org/10.1016/j.acra.2013.02. 011

22. Ingeman ML, Christensen MB, Bro F et al (2015) The Danish cancer pathway for patients with serious non-specific symptoms and signs of cancer-a cross sectional study of patient characteristics and cancer probability. BMC Cancer 15:421. https://doi.org/10. 1186/s12885-015-1424-5

23. van Ommen F, de Jong HWAM, Dankbaar JW et al (2019) Dose of CT protocols acquired in clinical routine using a dual-layer detector CT scanner: a preliminary report. Eur J Radiol 112:65-71. https:// doi.org/10.1016/j.ejrad.2019.01.011

24. van Ommen F, Bennink E, Vlassenbroek A et al (2018) Image quality of conventional images of dual-layer SPECTRAL CT: a phantom study. Med Phys 45:3031-3042. https://doi.org/10.1002/ mp.12959

25. Harris PA, Taylor R, Thielke R et al (2009) Research electronic data capture (REDCap) - a metadata-driven methodology and workflow process for providing translational research informatics support. J Biomed Inform 42:377-381. https://doi.org/10.1016/j.jbi.2008.08. 010

26. Hounsfield GN (1973) Computerized transverse axial scanning (tomography). 1. Description of system. Br J Radiol 46:1016-1022. https://doi.org/10.1259/0007-1285-46-552-1016

27. Petersilka M, Bruder H, Krauss B et al (2008) Technical principles of dual source CT. Eur J Radiol 68:362-368. https://doi.org/10. 1016/j.ejrad.2008.08.013

28. Megibow AJ, Sahani D (2012) Best practice: implementation and use of abdominal dual-energy CT in routine patient care. Am J Roentgenol 199:S71-S77. https://doi.org/10.2214/AJR.12.9074

29. Ho LM, Yoshizumi TT, Hurwitz LM et al (2009) Dual energy versus single energy MDCT: measurement of radiation dose using adult abdominal imaging protocols. Acad Radiol 16:1400-1407. https://doi.org/10.1016/j.acra.2009.05.002

30. Henzler T, Fink C, Schoenberg SO et al (2012) Dual-energy CT: radiation dose aspects. Am J Roentgenol 199:S16-S25. https://doi. org/10.2214/AJR. 12.9210

31. Jia JB, Houshyar R, Verma S et al (2016) Prostate cancer on computed tomography: a direct comparison with multi-parametric magnetic resonance imaging and tissue pathology. Eur J Radiol 85:261267. https://doi.org/10.1016/j.ejrad.2015.10.013

32. Roussel B, Ouellet GM, Mohile SG, Dale W (2015) Prostate cancer in elderly men: screening, active surveillance, and definitive therapy. Clin Geriatr Med 31:615-629. https://doi.org/10.1016/j.cger. 2015.07.004

33. Chen A-L, Liu A-L, Wang S et al (2015) Detection of gallbladder stones by dual-energy spectral computed tomography imaging. World J Gastroenterol 21:9993-9998. https://doi.org/10.3748/wjg. v21.i34.9993

Publisher's note Springer Nature remains neutral with regard to jurisdictional claims in published maps and institutional affiliations. 